\title{
BMJ Open Description of symptom course in a telemedicine monitoring clinic for acute symptomatic COVID-19: a retrospective cohort study
}

\author{
James B O'Keefe (D) , ${ }^{1}$ Elizabeth J Tong (D) , ${ }^{1}$ Ghazala D O'Keefe (D) , ${ }^{2}$ \\ David C Tong (i) ${ }^{3}$
}

To cite: O'Keefe JB, Tong EJ, 0 'Keefe GD, et al. Description of symptom course in a telemedicine monitoring clinic for acute symptomatic COVID-19: a retrospective cohort study. BMJ Open 2021;11:e044154. doi:10.1136/ bmjopen-2020-044154

- Prepublication history and additional material for this paper are available online. To view these files, please visit the journal online (http://dx.doi. org/10.1136/bmjopen-2020044154).

Received 24 August 2020 Revised 03 February 2021 Accepted 19 February 2021

Check for updates

(C) Author(s) (or their employer(s)) 2021. Re-use permitted under CC BY-NC. No commercial re-use. See rights and permissions. Published by BMJ.

${ }^{1}$ Division of General Internal Medicine, Department of Medicine, Emory University School of Medicine, Atlanta, Georgia, USA

${ }^{2}$ Section of Vitreoretinal Surgery and Diseases, Section of Uveitis and Vasculitis, Department of Ophthalmology, Emory University School of Medicine, Atlanta, Georgia, USA

${ }^{3}$ Division of Hospital Medicine, Department of Medicine, Emory University School of Medicine, Atlanta, Georgia, USA

Correspondence to Dr James B O'Keefe; jbokeef@emory.edu

\section{ABSTRACT}

Objective Describe the disease course in a cohort of outpatients with COVID-19 and evaluate factors predicting duration of symptoms.

Design Retrospective cohort study.

Setting Telemedicine clinic at a large medical system in Atlanta, Georgia.

Participants 337 patients with acute COVID-19. Exclusion criteria included intake visit more than 10 days after symptom onset and hospitalisation prior to intake visit. Main outcome measures Symptom duration in days. Results Common symptoms at intake visit are upper respiratory $(73 \%$ cough, $55 \%$ loss of smell or taste, $57 \%$ sinus congestion, $32 \%$ sore throat) and systemic $(66 \%$ headache, $64 \%$ body aches, $53 \%$ chills, $30 \%$ dizziness, $36 \%$ fever). Day of symptom onset was earliest for systemic and upper respiratory symptoms (median onset day 1 for both), followed by lower respiratory symptoms (day $3,95 \% \mathrm{Cl} 2$ to 4), with later onset of gastrointestinal symptoms (day 4, 95\% Cl 3 to 5), when present. Cough had the longest duration when present with median 17 days $(95 \% \mathrm{Cl} 15$ to 21$)$, with $42 \%$ not resolved at final visit. Loss of smell or taste had the second longest duration with 14 days $(95 \% \mathrm{Cl} 12$ to 17$)$, with $38 \%$ not resolved at final visit. Initial symptom severity is a significant predictor of symptom duration ( $p<0.01$ for multiple symptoms). Conclusions COVID-19 illness in outpatients follows a pattern of progression from systemic symptoms to lower respiratory symptoms and persistent symptoms are common across categories. Initial symptom severity is a significant predictor of disease duration for most considered symptoms.

\section{INTRODUCTION}

COVID-19 has brought large numbers of patients to medical attention within a span of months for care of a previously undescribed illness. Early reports on the presentation and natural history of COVID-19 appropriately focused attention on the severe cases and critically ill. ${ }^{1-5}$ Subsequent surveillance has demonstrated that the majority of patients have milder forms of illness ${ }^{6}$ and it is recommended that they remain at home with
Strengths and limitations of this study

By systematically calling patients throughout acute illness, we are able to provide a visual representation of symptoms of acute illness in outpatients.

- Missing data are minimal during acute illness as patients are followed until symptom improvement.

- We used standardised templates for all patients and are able to analyse predictors of symptom duration for specific variables including age, comorbidities and symptom severity.

- We are a single-centre study with limited patient numbers.

- We do not follow patients until disease resolution and cannot define an end date for all symptoms.

medical supervision. ${ }^{78}$ Although the duration of home isolation is defined based on symptoms, ${ }^{7}$ understanding of the symptom course of outpatients with COVID-19 is limited and most reports include presenting symptoms alone or cross-sectional follow-up information. ${ }^{9-18}$ Predictors of individual symptom duration have not been described.

In March 2020, we established a virtual clinic for the care of patients in home isolation with COVID-19: the 'Virtual Outpatient Management Clinic' (VOMC), using available knowledge for assessment and treatment guidelines. All patients underwent VOMC intake visits with a physician or advanced practice provider (APP), including assessment of specific COVID-19 symptoms using a standardised clinical note. Patients were followed for symptom management with regular telephone calls by registered nurses (RNs) and APPs until improvement or hospitalisation. Subsets from this cohort have been reported elsewhere in a small case series ${ }^{19}$ and for hospitalisation risk prediction ${ }^{20}$; the current study is the first to analyse complete longitudinal symptom reporting for the cohort. 
As it became clear in clinical practice that symptom duration varies substantially between patients, we undertook this study to determine the predictors of the symptom course of our VOMC cohort. We hypothesised that a combination of demographics, comorbidities and initial symptom severity would predict symptom duration.

\section{METHODS}

\section{Study setting}

The study was a retrospective cohort study, conducted at Emory Healthcare, the largest academic health system in Georgia (serving the greater Atlanta metropolitan area), which includes more than 250 provider locations and 120 primary care locations. The VOMC comprised an intake team of 14 physicians and 3 APPs from two primary care clinics; and follow-up call teams included 19 redeployed RNs and 20 APPs. All intake providers were trained in the use of the risk assessment tool in a 1-hour webinar and conducted a median of 25 intake visits during the study period (range: 5-99), with the majority of intake visits conducted by physicians $(83.6 \%)$.

\section{Study cohort}

We included outpatient adults who completed their VOMC intake visit between 24 March 2020 and 26 May 2020 with initial symptom dates between 17 March and 20 May. We excluded patients hospitalised prior to the intake visit and patients with an intake visit occurring more than 10 days after symptom onset in order to improve the accuracy of early symptom reporting.

During the study period, outpatient COVID-19 testing was conducted by medical providers using nasopharyngeal sampling for real-time reverse transcription-PCR (RT-PCR) detection of SARS-CoV-2. Testing of outpatients occurred primarily at a screening clinic (converted outpatient clinical space) and did not include a clinical assessment except for triage of visibly unstable patients. As test volume increase in April 2020, a drive-through site was added (accounting for 27\% of VOMC referrals during the study period). Patients requiring in-person evaluation could be triaged at any time in their illness to the emergency department (ED) or a lower acuity 'in-person' Acute Respiratory Clinic (ARC), described elsewhere. ${ }^{21}$ During the study period, $12 \%$ of VOMC patients were seen in the ARC (either for initial diagnosis or evaluation of symptoms).

Adult patients with positive RT-PCR results from the outpatient sites or EDs were called by a result notification team to provide isolation advice and refer to the VOMC. All patients with positive RT-PCR were offered VOMC referral during the study period. The criteria for testing and details of care are outlined in box 1. During the VOMC intake visit, symptom severity was assessed by the provider using criteria in box 1 as well as self-reported by the patient.

\section{Data sources}

Study data were obtained from two specific provider note types deployed in March 2020 within the Emory
Box 1 Virtual Outpatient Management Clinic (VOMC) care during study period

Outpatient COVID-19 testing criteria (March-April 2020):

1. Symptom(s): either (a) fever, cough or shortness of breath, or (b) two symptoms from the following: sore throat, congestion, myalgias, fatigue, diarrhoea, loss of smell.

2. Prioritise: (a) frontline healthcare workers, (b) students on campus and health professions, (c) Centers for Disease Control and Prevention employees, (d) patients with risk factors (age, comorbidity, immunosuppression, work in a communal setting).

3. Setting: outpatient clinic repurposed as testing site (12 March 2020) and additional drive-through site added to expand capacity (9 April 2020).

\section{Emergency department COVID-19 testing criteria (March} 2020):

1. Symptom(s): cough, fever, sore throat or shortness of breath.

2. Prioritise: (a) severe illness (difficulty breathing or other indication for admission), (b) high-risk comorbidities including chronic lung disease, heart disease, chronic kidney disease, diabetes, immunocompromising conditions, or (c) communal housing or living with high-risk individual.

\section{Acute Respiratory Clinic in-person care site (April-May} 2020):

1. Referrals for evaluation (and testing if needed) from sources: COVID-19 hotline (triage of incoming patient calls), physician offices and VOMC for in-person evaluation of VOMC patients.

2. Testing criteria: none specified, at provider discretion only.

3. Setting: primary care clinic repurposed as acute care site for respiratory complaints (known or possible COVID-19), with services including phlebotomy, plain radiography, ECG, pulse oximetry. Staffed daily by one to two physicians and advanced practice providers from the general internal medicine and one infectious disease specialist.

\section{VOMC enrolment criteria:}

1. Diagnosis of COVID-19 by nasopharyngeal PCR.

2. Requesting* outpatient monitoring and/or management of COVID-19 symptoms.

3. Able to complete telemedicine intake (synchronous audio/video connection by smartphone or computer preferred), with telephoneonly visit as backup option.

\section{Intake VOMC visit:}

1. Documentation template includes symptom history, symptom severity (patient-reported and provider-assessed), medical history, physical examination and risk assessment.

2. Symptoms assessed: 'systemic' (fever, chills, body aches, dizziness, headache, joint pain), 'upper respiratory' (loss of smell or taste, sinus congestion, sore throat, cough), 'lower respiratory' (chest tightness, shortness of breath with exertion, shortness of breath at rest, wheezing), 'gastrointestinal' (abdominal pain, nausea, diarrhoea), as well as confusion and rash. Note: symptoms assessed as a single list and not grouped into categories during assessment.

3. Provider gives advice for (1) symptom management, (2) home isolation guidance and (3) outpatient monitoring.

Provider-assessed symptom severity definition (at VOMC intake visit):

1. Mild

a. Respiratory: cough, sputum production. 


\section{Box 1 Continued}

b. Systemic: fever, chills, malaise, myalgia, anorexia, diarrhoea, vomiting, headache.

2. Moderate

a. Respiratory: severe cough, dyspnoea on exertion, wheezing or sensation of mid-chest tightness.

b. Systemic: N/A (not provided in VOMC clinical guideline).

3. Severe

a. Resting dyspnoea, laboured breathing, resting pulse oximetry $\leq 92 \%$, pleuritic pain, haemoptysis.

b. Systemic: acute confusion, severe weakness, syncope, acute decline in functional status.

Follow-up phone calls (March-June 2020):

1. Patients receive VOMC follow-up telephone calls based on hospitalisation risk tool ${ }^{20}$ that includes age, comorbidity, symptom severity and social support:

a. Low risk: every other day for a minimum of 7 days from symptom onset.

b. Intermediate risk: daily for a minimum of 14 days from symptom onset.

c. High risk: twice daily for a minimum of 21 days from symptom onset.

2. All patients called until the intervals above and for a minimum 3 days after improvement in fevers (without antipyretics) and improvement in respiratory symptoms (whichever criterion was longer).

3. Patients with improving or worsening symptoms could change risk level after enrolment at provider discretion.

*All patients with positive results were notified by telephone and offered VOMC referral.

Healthcare electronic health record (Cerner Corp, Kansas City, Missouri, USA): (1) VOMC provider intake visit and (2) VOMC follow-up telephone call. The intake visit assessment note template included (1) documentation of specific COVID-19 symptoms including onset and offset dates, (2) patient-reported and providerassessed symptom severity, and (3) documentation of specific medical conditions associated with risk of severe COVID-19 (based on medical literature search in March 2020). The VOMC follow-up telephone call template included an identical symptom list with 'yes/no' selection for documentation of the presence or absence of symptoms at follow-up.

If symptom onset date was not identified in VOMC notes, we conducted manual chart review of telephone records prior to VOMC enrolment. Additional demographic information including age, gender and race (if recorded) was included from the electronic health record.

To ensure that symptoms were counted only once a day per patient among patients receiving two calls per day, if a symptom was listed as present more than once for a particular day, it was counted only once. Among patients receiving calls every other day, if a symptom was present on both the preceding and subsequent days, it was listed as present on the single non-call day in between for symptom duration.

\section{Main outcomes}

To create a visual representation of overall disease as a heat map, we define day 1 as the first day a patient had any symptom and each individual symptom is counted only on days present.

The main outcome was duration in days for each specific symptom, using the first and last documented dates a symptom was present. Because patients could be discharged from VOMC with ongoing symptoms, if a symptom was present on the last nurse phone call it was considered censored for survival analysis. If a symptom was not present on the last nurse phone call, then the symptom was considered resolved.

The secondary outcome was the day of symptom onset. Symptoms were grouped into systems: upper respiratory (cough, congestion, sore throat, loss of smell or taste), systemic (fever, body aches, chills, dizziness, headache, joint pain), lower respiratory (shortness of breath (SOB) with exertion, SOB at rest, chest tightness, wheezing) and gastrointestinal (nausea, abdominal pain, diarrhoea). Confusion and rash were not included into symptom groups. For initial symptom severity, we used the provider-assessed severity at the intake visit (criteria listed in box 1). If provider-assessed severity was not available $(n=25)$, then the patient-reported severity at intake visit was used ( $n=18$ mild and $n=7$ moderate). Clinical record extraction was conducted on 21 June 2020 at which time all enrolled patients had at least 30 days of follow-up based on symptom start date and all patients had received their final VOMC nurse call.

\section{Potential bias}

Testing criteria are noted in box 1 . Healthcare employees were prioritised in the outpatient screening process and may be over-represented in the cohort. Testing in the ED prioritised patients with more severe symptoms and likely under-represented mild disease. Furthermore, patient enrolment in VOMC was voluntary at the time of results notification, which may result in selection bias. Patients were scheduled for the minimum recommended follow-up calls at the time of intake (and could later extend care further if needed) but could disengage on request, which could lead to attrition bias.

\section{Predictors}

Demographics, comorbidities and initial symptom severity were tested as predictors of symptom duration.

\section{Statistical analysis}

Survival analysis was used to analyse symptom start date by system and duration of individual symptoms. Kaplan-Meier curves were constructed for symptom onset (grouped by systems) to calculate median day of onset with pairwise log-rank test used to compare the system groupings. Kaplan-Meier curves were also used to determine the median duration for each symptom. Cox proportional hazard models were constructed but the proportional hazards requirement was not met for 
several covariate symptom combinations so Cox models were not used. Time-varying covariates can be included as strata but different baseline hazards are modelled for each strata so the effect of the strata covariate is not estimated. ${ }^{22}$ Cox models with time-by-covariate interactions are difficult to interpret and are included in the supplement (online supplemental table 1).

Subsequent analysis showed accelerated failure time (AFT) models had a better fit. AFT models are an alternate method of survival analysis which is parametric and does not require proportional hazards. To decrease the chance of false positive findings, we screened each comorbidity to see if it was a significant predictor of symptom duration with symptom duration analysed as strata (online supplemental table 2). Models were developed for each symptom including the covariates that were significant (gender, initial symptom severity, asthma, immunosuppression, obesity) to the $\mathrm{p}<0.001$ level and race. Statistically significant covariates for each symptom's model were retained in the final AFT models. The Akaike information criterion showed the AFT models were a better fit than the Cox proportional hazards models (online supplemental table 3). Log-normal and log-logistic distribution AFT models appeared to fit the data the best with very similar results (online supplemental table 4), and we present the log-logistic distribution model in this paper with projected survival curves for different models in online supplemental figure 1. Goodness-of-fit testing was performed for the log-normal and log-logistic AFT models (online supplemental figure 2). Self-reported symptom severity was used if provider-assessed symptom severity was missing $(n=25)$. This resulted in a similar goodness of fit compared with imputing the missing provider-assessed severity (online supplemental table 5). Statistical analysis was performed using RStudio V.4.0.3 packages survival and flexsurv (R core Team, 2020).

\section{Patient and public involvement}

Patients and the public were not involved in the design and conduct of the study, outcomes, recruitment or planned dissemination.

\section{RESULTS}

There were 551 intake visits completed in the VOMC between 24 March 2020 and 26 May 2020. We included 337 patients in the study after excluding: 198 patients with VOMC intake visit more than 10 days after symptom onset, 6 patients without documented positive RT-PCR test for SARS-CoV-2, 3 patients hospitalised prior to VOMC enrolment, 3 patients with blank or uninterpretable symptom entries and 4 patients with neither provider nor self-reported initial symptom severity. Of the included patients, $33(10 \%)$ were subsequently hospitalised and 7 of the hospitalised patients resumed VOMC care after hospital discharge.

The testing location for included patients was primarily outpatient $(n=304,90 \%)$, followed by ED $(n=33,10 \%)$.
During the study period (testing dates 15 March 2020-22 May 2020), the following number of patients tested positive for SARS-CoV-2 by RT-PCR at Emory Healthcare: 730 in the outpatient setting, 170 in the ED, 740 in the inpatient setting, 1 in ambulatory surgery and 1 patient in hospice.

\section{Characteristics of the study population}

Table 1 describes demographics, comorbidities and symptoms recorded at the VOMC intake visit for the cohort grouped by initial symptom severity. Our study population had a mean age of 45.7 years, $68 \%$ women and $52 \%$ black. The mean number of days from symptom onset to VOMC intake visit was 5.8 days, with follow-up phone calls continuing until mean symptom day 19 . There was a significant difference in the initial symptom severity by patient age. Asthma, heart failure and hypertension were significantly different $(\mathrm{p}<0.05)$ between the initial symptom severity groups. Only eight patients had severe initial symptoms severity and four (50\%) were hospitalised during care. Of the four non-hospitalised patients in the severe symptom group, two were evaluated by the ARC and determined to be stable for outpatient monitoring and two were managed by the VOMC telemedicine team alone without escalation to in-person care.

\section{Symptoms at VOMC intake visit}

The most frequently reported symptoms occurring prior to and at the time of the VOMC intake visit included: $73 \%$ cough, $66 \%$ headache, $64 \%$ body aches, $57 \%$ sinus congestion, $55 \%$ loss of smell or taste and 53\% chills (table 1). All symptoms within the systemic, lower respiratory and gastrointestinal systems were significantly different between severity groups. The only symptoms that were not significant were headache, sinus congestion, sore throat and rash.

\section{Time course of individual symptoms}

Figure 1A displays heat maps of symptoms for 304 patients, presenting the daily percentage of patients with specified symptoms over a 30-day follow-up period. The 33 patients hospitalised after VOMC intake are not included in figure 1 because daily symptom data were not collected during hospitalisations. Among symptoms included in this visual, the highest daily prevalence reported during 30 days of follow-up was for cough $(60 \%)$, loss of smell or taste $(50 \%)$, sinus congestion $(46 \%)$, body aches $(46 \%)$ and headache $(45 \%)$. The most frequent remaining symptoms at 30 days were cough (5\%), body aches (4\%), sinus congestion (3\%) and SOB with exertion (3\%). Fever was not a prominent symptom during 30 days of follow-up, with peak daily prevalence of $22 \%$ in the first 2 days of illness.

\section{Time course of symptoms by initial symptom severity}

The heat map findings for mild initial symptom severity group ( $\mathrm{n}=209$ for heat map) demonstrate similar rates of initial upper respiratory symptoms compared with the entire heat map cohort, with peak daily prevalence of 
Table 1 Demographics, comorbidities and symptoms at VOMC intake visit

Initial symptom severity

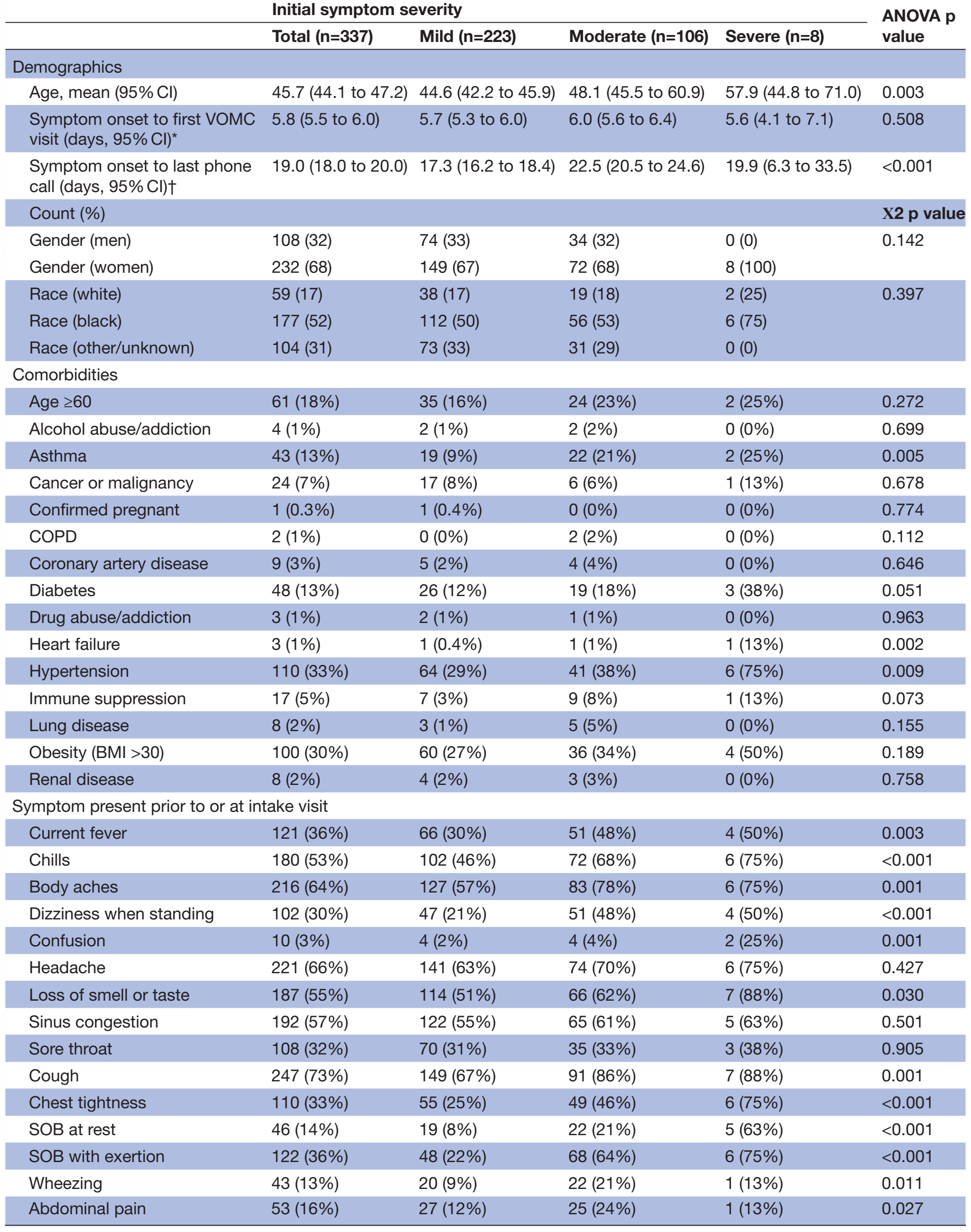

Continued 
Table 1 Continued

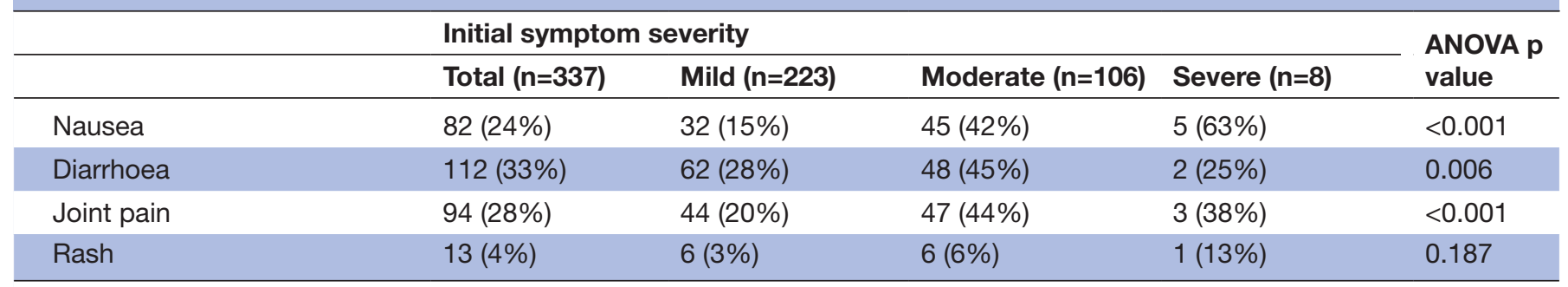

${ }^{*}$ Number of days from initial symptom(s) of COVID-19 to completion of telemedicine intake visit for the VOMC, inclusive of time required for testing, result notification and scheduling with VOMC.

†Number of days from initial symptom(s) of COVID-19 to the final telephone call with VOMC. The calls would end with patient-reported symptom improvement (not necessary resolution) or hospital admission.

ANOVA, analysis of variance; BMI, body mass index; COPD, chronic obstructive pulmonary disease; SOB, shortness of breath; VOMC, Virtual Outpatient Management Clinic.

cough in $52 \%$ and sinus congestion in $45 \%$ during the first week (figure 1B). Rates of lower respiratory symptoms were lower (eg, SOB with exertion in $20 \%$ on day 8 , vs $30 \%$ in overall cohort). Rates of persistent symptoms at 30 days were lower in the mild initial symptom severity cohort, with no more than $1 \%$ reporting persistence of each individual symptom.

The heat map for moderate initial symptom severity (figure 1C, $\mathrm{n}=91$ ) had higher rates of all symptoms (compared with overall group and mild initial symptom severity). Differences were less for upper respiratory symptoms such as sinus congestion (peak prevalence $54 \%$ in moderate group compared with $46 \%$ overall) and greater for lower respiratory symptoms (eg, SOB with exertion $56 \%$ vs $30 \%$ ), systemic symptoms (eg, joint pain $36 \%$ vs 18\%) and gastrointestinal symptoms (eg, diarrhoea $36 \%$ vs $21 \%$ ). At 30 days the most frequent symptoms remaining were cough $(9 \%)$, sinus congestion $(8 \%)$, body aches $(8 \%)$, joint pain $(6 \%)$, loss of taste or smell (5\%), SOB with exertion (5\%) and headache (4\%).

There were too few non-hospitalised patients with severe initial symptom severity $(n=4)$ to represent with a heat map. This group had high prevalence $(100 \%$ within the first week) of chills, body aches, loss of smell or taste, sore throat, cough, chest tightness and $\mathrm{SOB}$ with exertion. At 30 days, three patients $(75 \%)$ still had cough and SOB with exertion.

\section{Timing of symptom onset by system}

The median day of symptom onset determined by KaplanMeier curves is shown in table 2. The onset for systemic and upper respiratory symptoms frequently occurred on day 1 , with gastrointestinal and lower respiratory symptoms occurring later. Recognising that cough can be a manifestation of upper airway or lower airway infections, we analysed cough separately, finding a median start of 1 day (95\% CI 1 to 1 ). This was not different from the median start day for upper respiratory symptoms $(p=0.253)$, but it was significantly different than the median start day for lower respiratory symptoms $(\mathrm{p}<0.001)$ so we grouped cough with the upper respiratory system.

\section{Duration of each symptom}

Table 3 describes the median days and 95\% CI for each symptom obtained from Kaplan-Meier survival curves, censoring symptoms if present at the final VOMC phone call. When present, cough had the longest duration with 17 days (95\% CI 15 to 21), followed by loss of smell or taste with 14 days (95\% CI 12 to 17) and sinus congestion with 13 days (95\% CI 11 to 15). SOB with exertion, when present, lasted 12 days (95\% CI 10 to 16 ).

Patients reporting improving symptoms could be discharged from VOMC with symptoms present. The percentage of patients reporting resolution of each symptom is presented in table 4 . The symptoms most frequently unresolved at the time of the final phone call were cough (115 of 276 patients with cough, 42\%), loss of smell or taste (89 of 232, 38\%), sinus congestion (89 of $244,36 \%)$ and SOB with exertion (65 of $188,35 \%)$.

\section{Symptom duration predicted by covariates}

Table 5 presents the results of the final AFT models with log-logistic distribution fitted individually for each symptom. The AFT duration multiplier is the factor by which the survival time is multiplied for that group compared with the reference group. For example, obesity was the only significant predictor of joint pain duration with 1.74-fold (95\% CI 1.09 to 2.76) longer duration, which is $74 \%$ (95\% CI $9 \%$ to $176 \%$ ) longer symptoms than those without obesity. Asthma was the only significant predictor for wheezing, increasing duration by $166 \%$ (95\% CI $39 \%$ to $408 \%$ ). Asthma and black race were both predictors of duration for SOB at rest, with asthma increasing SOB duration by $97 \%$ (95\% CI $20 \%$ to $224 \%$ ) and black race by $178 \%$ (95\% CI $63 \%$ to $407 \%$ ). Diarrhoea is more complex. Patients with moderate initial symptom severity had $72 \%$ longer $(95 \%$ CI $24 \%$ to $138 \%$ ) duration of diarrhoea than those with mild initial symptom severity, while black patients had a $44 \%(95 \%$ CI $-63 \%$ to $-16 \%$ ) shorter duration of diarrhoea. Initial symptom severity (from VOMC intake visit) was a significant predictor for over half of our symptoms: body aches, chest tightness, chills, congestion, cough, diarrhoea, 


\begin{tabular}{|c|c|c|c|c|c|c|c|c|c|c|c|c|c|c|c|c|c|c|c|c|c|c|c|c|c|c|c|c|c|c|}
\hline Symptom & 1 & 2 & 3 & 4 & 5 & 6 & 7 & 8 & 9 & 10 & 11 & 12 & 13 & 14 & 15 & 16 & 17 & 18 & 19 & 20 & 21 & 22 & 23 & 24 & 25 & 26 & 27 & 28 & 29 & 30 \\
\hline current fever & 22 & 22 & 21 & 20 & 18 & 18 & 16 & 14 & 11 & 8 & 10 & 9 & 5 & 3 & 2 & 1 & 1 & 0 & 1 & 0 & 0 & 0 & 0 & 0 & 0 & 0 & 0 & 0 & 0 & \\
\hline chills & 27 & 34 & 35 & 34 & 31 & 29 & 26 & 19 & 18 & 12 & 10 & 9 & 6 & 5 & 5 & 4 & 7 & 1 & 1 & 0 & 1 & 0 & 1 & 1 & 1 & 1 & 1 & 1 & 1 & \\
\hline body & 37 & 43 & 45 & 46 & 42 & 37 & 35 & 34 & 32 & $26 \mid$ & 23 & 21 & 19 & 16 & 14 & 11 & 9 & 7 & 8 & 7 & 7 & 7 & 6 & 7 & 6 & 6 & 5 & 4 & 4 & \\
\hline dizzir & 5 & 11 & 12 & 15 & 15 & 16 & 14 & 15 & 15 & 14 & 13 & 11 & 10 & 10 & 7 & 5 & 6 & 5 & 3 & 3 & 2 & 2 & 3 & 2 & 2 & 1 & 1 & 0 & 1 & \\
\hline confu & 0 & 0 & 0 & 0 & 0 & 0 & 0 & 0 & 0 & 0 & 0 & 0 & 0 & 0 & 0 & 0 & 0 & 0 & 0 & 0 & 0 & 0 & 0 & 0 & 0 & 0 & 0 & 0 & 0 & \\
\hline nead; & 37 & 44 & 45 & 43 & 42 & 38 & 35 & 33 & 29 & 27 & 25 & 23 & 20 & 19 & 17 & 14 & 12 & 11 & 10 & 10 & 8 & 8 & 9 & 8 & 5 & 7 & 6 & 4 & 4 & \\
\hline oss C & 22 & 30 & 37 & \begin{tabular}{|l|}
42 \\
\end{tabular} & 48 & 50 & 50 & 50 & 48 & 43 & 41 & 33 & 29 & 27 & 25 & 18 & 20 & 16 & 13 & 12 & 10 & 9 & 8 & 8 & 7 & 7 & 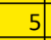 & 4 & 3 & \\
\hline inus & 32 & 37 & 43 & 45 & 46 & 44 & 45 & 42 & 40 & 38 & 37 & 32 & 29 & 25 & 25 & 21 & 21 & 19 & 16 & 12 & 12 & 11 & 8 & 8 & 7 & 6 & 5 & 4 & 4 & \\
\hline ore $t$ & 21 & 24 & 23 & 22 & 20 & 17 & 16 & 14 & 13 & 10 & 10 & 7 & 7 & 7 & 7 & 6 & 6 & 6 & 6 & 3 & 3 & 3 & 2 & 3 & 3 & 2 & 1 & 2 & 1 & \\
\hline ough & 46 & 53 & 55 & 57 & 60 & 57 & 56 & 56 & 56 & $52 \mid$ & 47 & 41 & 39 & 36 & 34 & 30 & 30 & 25 & 21 & 20 & 18 & 18 & 14 & 14 & 12 & 10 & 0 & 7 & 7 & \\
\hline hest & 11 & 15 & 18 & 21 & 22 & 23 & 25 & 23 & 22 & 20 & 19 & 18 & 14 & 12 & 10 & 10 & 9 & 7 & 5 & 4 & 5 & 4 & 4 & 3 & 3 & 4 & 3 & 2 & 3 & \\
\hline shortr & 4 & 4 & 5 & 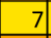 & 7 & 7 & 7 & 7 & 7 & 5 & 5 & 5 & 3 & 3 & 3 & 2 & 1 & 2 & 1 & 0 & 0 & 0 & 0 & 1 & 0 & 1 & 0 & 0 & 0 & \\
\hline shortr & 13 & 17 & 20 & 21 & 23 & 26 & 29 & 30 & 30 & $27 \mid$ & 25 & 25 & 22 & 22 & 20 & 20 & 16 & 14 & 13 & 13 & 11 & 10 & 9 & 8 & 7 & 8 & 8 & 5 & 6 & \\
\hline whee & 3 & 4 & 5 & 6 & 6 & 7 & 7 & 6 & 7 & 6 & 5 & 4 & 2 & 1 & 0 & 1 & 1 & 1 & 0 & 1 & 0 & 1 & 1 & 0 & 1 & 0 & 1 & 1 & 1 & \\
\hline abdon & 3 & 4 & 6 & 7 & 10 & 10 & 7 & 7 & 7 & 7 & 5 & 3 & 2 & 3 & 3 & 2 & 2 & 1 & 1 & 0 & 2 & 1 & 2 & 1 & 0 & 1 & 0 & 0 & 0 & \\
\hline nausea & 6 & 7 & 8 & 10 & 12 & 13 & 13 & 13 & 14 & \begin{tabular}{|l|}
12 \\
\end{tabular} & 11 & 9 & 7 & 8 & 7 & 6 & 3 & 3 & 3 & 2 & 3 & 3 & 3 & 2 & 1 & 1 & 1 & 1 & 0 & \\
\hline diarrhe & 12 & 15 & 15 & \begin{tabular}{|l|}
18 \\
\end{tabular} & 20 & 21 & 20 & 19 & 16 & 15 & 13 & 12 & 9 & 10 & 10 & 7 & 6 & 5 & 4 & 4 & 4 & 2 & 2 & 1 & 1 & 1 & 1 & 1 & 0 & \\
\hline joint $p$ & 9 & 13 & 15 & 17 & 18 & 18 & 17 & 14 & 14 & 12 & 13 & 14 & 12 & 11 & 10 & 8 & 7 & 7 & 6 & 5 & 4 & 5 & 4 & 4 & 3 & 3 & 3 & 3 & 3 & \\
\hline rash & 1 & 1 & 1 & 2 & 2 & 2 & 2 & 2 & 2 & 2 & 2 & 2 & 2 & 2 & 2 & 2 & 1 & 1 & 1 & 1 & 1 & 1 & 1 & 1 & 1 & 0 & 1 & 0 & & \\
\hline
\end{tabular}

Mild initial symptoms $=209$

\begin{tabular}{|c|c|c|c|c|c|c|c|c|c|c|c|c|c|c|c|c|c|c|c|c|c|c|c|c|c|c|c|c|c|c|}
\hline Symptom & 1 & 2 & 3 & 4 & 5 & 6 & 7 & 8 & \begin{tabular}{l|l}
9 & 1
\end{tabular} & \begin{tabular}{|l|l|}
10 \\
\end{tabular} & $11 \mathrm{1}$ & 12 & 13 & \begin{tabular}{|l|l|}
14 \\
\end{tabular} & 15 & 16 & 17 & 18 & \begin{tabular}{|l|l|}
19 \\
\end{tabular} & 20 & 21 & $22 \mid$ & 232 & 24 & 25 & $26 \mid$ & \begin{tabular}{|l|l}
27 \\
\end{tabular} & 28 & 29 & 30 \\
\hline current fever & 19 & 19 & 17 & 17 & 14 & 14 & 11 & 11 & 8 & 6 & 7 & 5 & 3 & 1 & 1 & 0 & 0 & 0 & 0 & 0 & 0 & 0 & 0 & 0 & 0 & 0 & 0 & 0 & 0 & 0 \\
\hline chills & 26 & 30 & 29 & 27 & 22 & 20 & 16 & 12 & 10 & 6 & 5 & 4 & 3 & 3 & 2 & 1 & 1 & 0 & 0 & 0 & 0 & 0 & 0 & 0 & 0 & 0 & 0 & 0 & 0 & 0 \\
\hline body aches & 34 & 38 & 38 & 36 & 31 & 26 & 23 & 22 & 20 & 18 & 16 & 13 & 13 & 9 & 7 & 6 & 4 & 3 & 3 & 3 & 2 & 4 & 3 & 3 & 2 & 2 & 2 & 1 & 1 & 1 \\
\hline dizziness when standing & 2 & 7 & 8 & 11 & 10 & 10 & 6 & 7 & 8 & 9 & 8 & 6 & 6 & 5 & 4 & 2 & 2 & 1 & 0 & 0 & 1 & 1 & 1 & 1 & 0 & 0 & 0 & 0 & 0 & 0 \\
\hline confusion & 0 & 0 & 0 & 0 & 0 & 0 & 0 & 0 & 0 & 0 & 0 & 0 & 0 & 0 & 0 & 0 & 0 & 0 & 0 & 0 & 0 & 0 & 0 & 0 & 0 & 0 & 0 & 0 & 0 & 0 \\
\hline headache & 33 & 40 & 39 & 36 & 35 & 32 & 29 & 25 & 21 & 20 & 20 & 14 & 12 & 13 & 11 & 10 & 7 & 6 & 7 & 6 & 5 & 5 & 6 & 4 & 3 & 4 & 4 & 2 & 2 & 1 \\
\hline loss of smell or taste & 21 & 27 & 34 & 38 & 44 & 46 & 45 & 44 & 43 & 38 & 36 & 27 & 24 & 23 & 21 & 14 & 15 & 11 & 9 & 8 & 7 & 6 & 5 & 5 & 4 & 5 & 3 & 1 & 1 & 0 \\
\hline sinus congestion & 31 & 36 & 43 & 45 & 44 & 43 & 40 & 37 & 34 & 32 & 33 & 25 & 24 & 21 & 18 & 17 & 14 & 13 & 9 & 7 & 6 & 6 & 5 & 4 & 4 & 2 & 2 & 1 & 1 & 1 \\
\hline sore throat & 19 & 24 & 22 & 22 & 19 & 15 & 12 & 11 & 10 & 9 & 10 & 6 & 5 & 4 & 4 & 4 & 5 & 5 & 6 & 2 & 2 & 2 & 1 & 1 & 1 & 0 & 0 & 0 & 0 & 0 \\
\hline cough & 40 & 47 & 48 & 49 & 52 & 50 & 47 & 46 & 47 & 43 & 38 & 31 & 29 & 27 & 24 & 22 & 21 & 18 & 13 & 11 & 11 & 11 & 9 & 7 & 6 & 5 & 4 & 1 & 3 & 1 \\
\hline chest tightness & 9 & 11 & 12 & 14 & 17 & 17 & 16 & 15 & 14 & 13 & 14 & 13 & 10 & 7 & 7 & 7 & 6 & 6 & 4 & 3 & 3 & 2 & 2 & 1 & 0 & 0 & 0 & 0 & 0 & 1 \\
\hline shortness of breat & 3 & 3 & 2 & 3 & 3 & 4 & 3 & 5 & 4 & 3 & 3 & 4 & 2 & 3 & 2 & 2 & 1 & 1 & 0 & 0 & 0 & 0 & 0 & 0 & 0 & 0 & 0 & 0 & 0 & 0 \\
\hline shortness of breath with exer & 5 & 8 & 10 & 11 & 12 & 15 & 17 & 20 & 18 & 16 & 15 & 14 & 13 & 13 & 11 & 13 & 9 & 8 & 8 & 7 & 6 & 5 & 5 & 4 & 3 & 3 & 3 & 1 & 2 & 1 \\
\hline wheezing & 2 & 2 & 2 & 3 & 4 & 5 & 4 & 3 & 4 & 4 & 4 & 4 & 1 & 1 & 0 & 1 & 0 & 0 & 0 & 0 & 0 & 0 & 0 & 0 & 0 & 0 & 0 & 0 & 0 & 0 \\
\hline abdominal pain & 3 & 1 & 3 & 5 & 7 & 7 & 4 & 5 & 4 & 5 & 3 & 2 & 1 & 2 & 2 & 0 & 1 & 0 & 0 & 0 & 0 & 0 & 0 & 0 & 0 & 0 & 0 & 0 & 0 & 0 \\
\hline nausea & 3 & 4 & 5 & 8 & 9 & 10 & 9 & 9 & 11 & 9 & 9 & 5 & 3 & 5 & 5 & 3 & 1 & 1 & 1 & 0 & 1 & 1 & 0 & 0 & 0 & 0 & 0 & 0 & 0 & 0 \\
\hline diarrhea & 10 & 11 & 10 & 13 & 14 & 15 & 13 & 14 & 10 & 11 & 8 & 7 & 6 & 8 & 7 & 3 & 2 & 1 & 0 & 0 & 1 & 0 & 0 & 0 & 0 & 0 & 0 & 0 & 0 & 0 \\
\hline joint pain & 7 & 9 & 10 & 11 & 12 & 11 & 8 & 7 & 6 & 6 & 6 & 8 & 7 & 7 & 6 & 5 & 3 & 3 & 2 & 3 & 2 & 3 & 2 & 2 & 1 & 1 & 1 & 1 & 1 & 0 \\
\hline rash & 0 & 1 & 0 & 1 & 2 & 2 & 1 & 1 & 1 & 1 & 1 & 2 & 2 & 2 & 2 & 1 & 1 & 1 & o & 0 & 0 & \begin{tabular}{|l|l} 
\\
\end{tabular} & & 0 & & \begin{tabular}{|l|}
0 \\
\end{tabular} & 1 & & & 0 \\
\hline
\end{tabular}

Moderate initial symptoms $n=91$

\begin{tabular}{|c|c|c|c|c|c|c|c|c|c|c|c|c|c|c|c|c|c|c|c|c|c|c|c|c|c|c|c|c|c|c|}
\hline Sy & $=$ & 2 & 3 & 4 & 5 & 6 & 7 & 8 & 9 & 10 & 11 & 12 & 13 & 14 & 15 & 16 & 17 & 18 & 19 & 20 & 21 & 22 & 23 & 24 & 252 & 26 & 27 & 28 & 29 & \\
\hline urrent fever & & 29 & 28 & 25 & 25 & 26 & 26 & 17 & 15 & 10 & 15 & 16 & 8 & t & 4 & 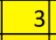 & 2 & 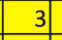 & & 3 & & & 4 & 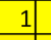 & 1 & 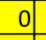 & & 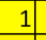 & 2 & \\
\hline hills & & 41 & 46 & 48 & 49 & 48 & 49 & 31 & 34 & 24 & 19 & 16 & 12 & 7 & & 7 & 5 & 2 & & 2 & & & 5 & 5 & 3 & 3 & & 4 & 5 & \\
\hline ody & 4 & 53 & 59 & 67 & 67 & 59 & 61 & 58 & 58 & 41 & 37 & 36 & 28 & 28 & 27 & 20 & 19 & 13 & 16 & 14 & 16 & 13 & 12 & 12 & 14 & 13 & 10 & 9 & 9 & \\
\hline izzir & 12 & 19 & 21 & 25 & 26 & 27 & 30 & 31 & 31 & 26 & 25 & 24 & 19 & 18 & 13 & a & 14 & 10 & 8 & 8 & 5 & & 5 & 3 & 5 & 2 & 1 & 1 & 1 & \\
\hline onfu & 1 & 1 & 0 & 0 & 0 & 0 & 3 & 2 & 1 & 0 & 0 & 0 & 2 & 1 & 1 & 0 & 1 & 0 & 0 & 0 & 0 & 4 & 0 & 0 & 0 & 0 & 0 & 0 & 7 & \\
\hline eada & 43 & 50 & 56 & 58 & 56 & 52 & 50 & 50 & 49 & 42 & 37 & 42 & 35 & 31 & 32 & 23 & 23 & 21 & 17 & 19 & 16 & 15 & 14 & 16 & 8 & 13 & 9 & 9 & 8 & \\
\hline losso & 21 & 36 & 41 & 50 & 54 & 57 & 60 & 62 & 59 & 50 & 50 & 45 & 39 & 32 & 30 & 25 & 26 & 26 & 18 & 19 & 16 & 16 & 14 & 14 & 10 & 12 & 9 & 9 & 6 & \\
\hline sinus & 35 & 41 & 43 & 45 & 49 & 46 & 54 & 51 & 53 & 50 & 45 & 47 & 39 & 36 & 39 & 29 & 36 & 31 & 30 & 24 & 26 & 23 & 15 & 18 & 13 & 16 & 13 & 10 & 10 & \\
\hline sore t & 23 & 24 & 23 & 21 & 19 & 19 & 20 & 17 & 18 & 10 & 9 & 8 & 8 & 14 & 13 & 9 & 6 & 7 & 5 & 3 & 4 & 6 & 4 & 9 & 7 & 7 & 4 & 5 & 3 & \\
\hline cough & 58 & 67 & 72 & 74 & 75 & 73 & 74 & 76 & 76 & 71 & 64 & 62 & 61 & 54 & 52 & 47 & 48 & 39 & 37 & 38 & 31 & 31 & 23 & 27 & 20 & 19 & 16 & 16 & 13 & \\
\hline chest & 14 & 21 & 28 & 31 & 30 & 34 & 42 & 39 & \begin{tabular}{|l|}
37 \\
\end{tabular} & 30 & 26 & 27 & 20 & 21 & 16 & 13 & 10 & 7 & 7 & 6 & 7 & 8 & 7 & 8 & 6 & 9 & 8 & 6 & 8 & \\
\hline shortr & 8 & 8 & 10 & 15 & 15 & 14 & 16 & 9 & 13 & 7 & 8 & 5 & 3 & 3 & 5 & 1 & F & 1 & 0 & 0 & 1 & 0 & 0 & 2 & 1 & 3 & 2 & 2 & 1 & \\
\hline shortr & 27 & 35 & 39 & 42 & 43 & 49 & 53 & 50 & 56 & 50 & 45 & 45 & 39 & 38 & 39 & 31 & 29 & 25 & 21 & 24 & 19 & 18 & 15 & 15 & 12 & 17 & 14 & 12 & 9 & \\
\hline whee & 5 & 8 & 10 & 13 & 10 & 12 & 15 & 15 & 14 & 10 & 8 & 5 & 4 & 3 & 3 & 3 & 3 & 3 & 2 & 2 & 3 & 5 & 5 & 2 & 3 & 2 & 3 & 2 & 2 & \\
\hline abdon & 4 & 9 & 13 & 12 & 17 & 15 & 13 & 12 & 14 & 12 & 10 & 6 & 4 & 6 & 1 & 6 & 5 & 4 & 5 & 1 & 5 & 4 & 6 & 5 & 2 & 4 & 2 & 2 & 0 & \\
\hline lause & 13 & 13 & 15 & 14 & 17 & 19 & 24 & 23 & 21 & 19 & 17 & 17 & 14 & 14 & 12 & 12 & 7 & 7 & 6 & 6 & 7 & 6 & 7 & 7 & 4 & 5 & 4 & 3 & 2 & \\
\hline tiarrh & 17 & 25 & 28 & 28 & 31 & 36 & 35 & 31 & 31 & 26 & 25 & 24 & 18 & 15 & 18 & 16 & 14 & 15 & 12 & 10 & 9 & 6 & 6 & 5 & 5 & 3 & 4 & 3 & 1 & \\
\hline oint & 14 & 21 & 28 & 31 & 32 & 32 & 36 & 31 & 30 & 25 & 28 & 28 & 21 & 21 & 19 & 16 & 17 & 16 & 15 & 9 & 8 & 6 & 4 & 7 & 6 & 8 & $y$ & 7 & 6 & \\
\hline rash & 3 & 3 & 3 & |7 & 3 & 2 & 4] & 3 & 3 & 2 & 4 & 2 & 1 & 1 & 2 & 2 & 7 & 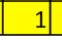 & 2 & 2 & 2 & 3 & 2 & 1 & 1 & 0 & 0 & 0 & & \\
\hline
\end{tabular}

Figure 1 Heat map of incidence of individual symptoms by illness day. (A) All patients, $n=304$ (\% of patients having symptom each day of COVID-19 disease); (B) mild provider-assessed symptom severity, $n=209$ and (C) moderate provider-assessed symptom severity, $\mathrm{n}=91$. 
Table 2 Median day of symptoms onset by system determined from Kaplan-Meier curves

$P$ value

\begin{tabular}{lllll} 
System & Median $(\mathbf{9 5} \% \mathbf{C l})$ & versus systemic & versus upper & $\begin{array}{l}\text { versus } \\
\text { lower }\end{array}$ \\
\hline Systemic & $1(1$ to 1$)$ & N/A & 0.032 & $<0.001$ \\
Upper & $1(1$ to 1$)$ & 0.032 & N/A & $<0.001$ \\
Lower & $3(2$ to 4$)$ & $<0.001$ & $<0.001$ & N/A \\
Gastrointestinal & $4(3$ to 5$)$ & $<0.001$ & $<0.001$ & 0.306
\end{tabular}

Systemic=fever, body aches, chills, dizziness, headache, joint pain; upper=cough, congestion, sore throat, loss of smell or taste; lower=shortness of breath with exertion, shortness of breath at rest, chest tightness, wheezing; gastrointestinal=nausea, abdominal pain, diarrhoea.

$\mathrm{N} / \mathrm{A}$, not available.

dizziness, fever, headache, nausea, SOB with exertion and sore throat (table 5). Moderate initial symptom severity increased symptom duration by an average of $63 \%$. Severe initial symptom severity increased symptom duration by an average of $260 \%$ compared with those with mild initial symptom severity. The residual plots to analyse goodness of fit for each symptom's model are in online supplemental figure 2 .

Table 3 Median duration of symptoms from Kaplan-Meier curves for all patients*

\begin{tabular}{lll}
\hline Symptom & $\begin{array}{l}\text { Number of } \\
\text { patients with } \\
\text { symptom }\end{array}$ & $\begin{array}{l}\text { Median } \\
\text { duration in } \\
\text { days (95\% Cl) }\end{array}$ \\
\hline Cough & 276 & $17(15$ to 21$)$ \\
\hline Loss of smell or taste & 232 & $14(12$ to 17$)$ \\
\hline Congestion & 244 & $13(11$ to 15$)$ \\
\hline SOB with exertion & 188 & $12(10$ to 16$)$ \\
\hline Body aches & 233 & $9(8$ to 10$)$ \\
\hline Chest tightness & 149 & $9(7$ to 12$)$ \\
\hline Headache & 258 & $9(7$ to 11$)$ \\
\hline Joint pain & 137 & $9(7$ to 11$)$ \\
\hline Rash & 33 & $9(6$ to 17$)$ \\
\hline Sore throat & 140 & $7(6$ to 9$)$ \\
\hline Nausea & 124 & $7(6$ to 8$)$ \\
\hline Current fever & 144 & $7(5$ to 8$)$ \\
\hline Diarrhoea & 168 & $6(5$ to 8$)$ \\
\hline Chills & 195 & $6(5$ to 7$)$ \\
\hline Wheezing & 70 & $6(4$ to 9$)$ \\
\hline Dizziness & 150 & $5(4$ to 7$)$ \\
\hline SOB at rest & 88 & $5(3$ to 6$)$ \\
\hline Abdominal pain & 100 & $4(2$ to 6$)$ \\
\hline Confusion & 20 & $2(1$ to 3$)$ \\
\hline
\end{tabular}

${ }^{*}$ Censoring symptoms if present at the final VOMC phone call. $\mathrm{SOB}$, shortness of breath; VOMC, Virtual Outpatient Management Clinic.

\section{DISCUSSION}

\section{Principal findings}

Whereas other studies have looked at cross-sectional analysis of COVID-19 symptoms, our study describes the longitudinal symptom course over the acute illness period for a telemedicine cohort, including the duration of reporting for each symptom and predictors of symptom duration.

We found that symptom onset starts with upper respiratory and systemic symptoms followed by lower respiratory and gastrointestinal symptoms. This is consistent with the fact that infected individuals produce large quantities of virus in the upper respiratory tract during the prodromal period. ${ }^{23}$ An important observation in this study is that initial symptom severity (at intake visit after test result) was a predictor for the symptom profile over time. Initial symptom severity predicts duration for the majority of symptoms, with significant associations for upper and lower respiratory symptoms as well as non-respiratory symptoms (including body aches, dizziness, chills, fever, headache, nausea and diarrhoea). Notable exceptions were joint pain and wheezing whose only predictors of symptom duration were the underlying risk factors of obesity and asthma, respectively. While these associations make sense clinically, an explanation for immunosuppression leading to longer duration of specific symptoms (abdominal pain and loss of smell or taste) is less intuitive. To decrease the chance of finding false positives, we fit models only for covariates that were significant predictors of overall symptom duration with symptoms analysed as strata, but some of the findings in the final models may be spurious. Nonetheless, the majority are very significant $(p<0.01)$ along with clinically significant changes in duration.

\section{Comparison with other studies}

Initial symptoms reported are similar to previous studies of mild COVID-19 and non-hospitalised subsets. ${ }^{12}{ }^{15}$ It differs from the overall reported literature, summarised in a systematic review of 148 studies. ${ }^{14}$ Notably, fever was less common $(n=121,36 \%)$ compared with $78 \%$ in the systematic review, while other symptoms are more common, for example: headache (65\% vs $13 \%)$, body 
Table 4 Symptoms unresolved at last phone call

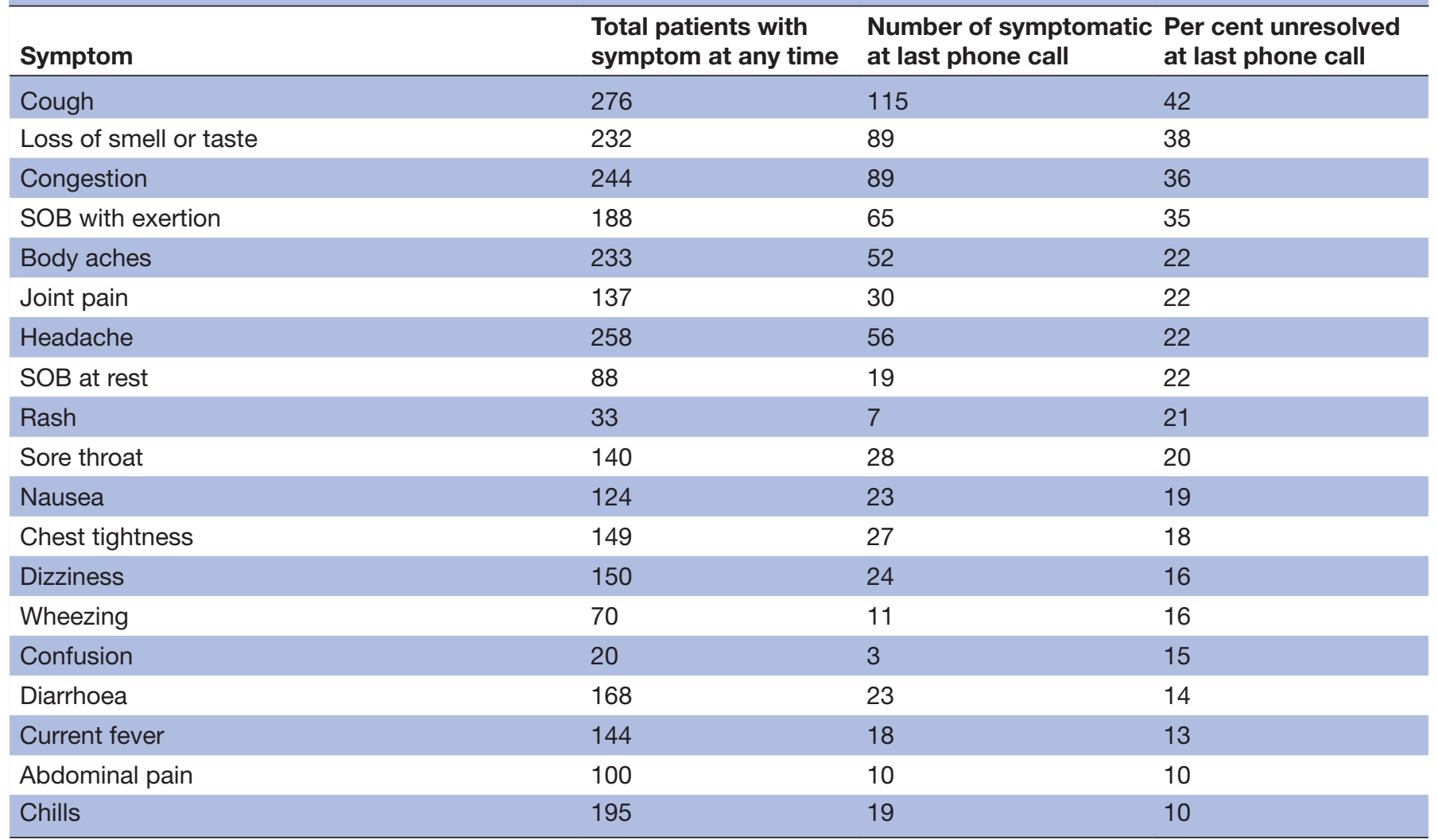

SOB, shortness of breath.

aches $(64 \%$ vs $17 \%)$ and hyposmia ( $55 \%$ vs $25 \%)$. The higher frequency of multiple symptoms may be due to our systematic approach to symptom inquiry. Rate of fever may be under-reported due to template wording 'current fever' but also may be less frequent in this cohort as increased testing availability has expanded the symptom profile of patients with 'mild COVID-19' eligible for testing (box 1).

We find that the visual course of illness and predictors of symptom duration have not been well described and this is an important contribution of this analysis. Narrative reviews have noted symptom progression similar to our report ${ }^{24}$ and the visual course reported here in heat map form illustrates the development of respiratory symptoms during and after the first week of illness among patients never requiring hospitalisation for COVID19. One longitudinal study of the first 10 days of illness included a follow-up call (day 30-45), reporting similar initial symptom profile (reporting loss of smell or taste, cough, fatigue and headache more common than fever) and persistent symptoms profile including loss of smell or taste, dyspnoea, cough and headache. ${ }^{18}$ Notably, the investigators found fatigue (not captured in our study) was prominent at acute and follow-up calls.

In our experience, many patients present for evaluation of non-resolving symptoms in the weeks that follow acute COVID-19. ${ }^{21}$ Reports have noted long duration of medical leave among persons with COVID-19, for example first responders in New York (leave duration mean 25.3 days, $\mathrm{SD}=13.2) .{ }^{25}$ We are able to differentiate the likelihood of prolonged symptoms in our cohort using mild and moderate initial symptom severity, which may aid in clinical counselling and anticipation of symptom recovery times. Given reports of delayed recovery of symptoms after hospitalisation, ${ }^{26}$ and in outpatients, ${ }^{19}$ the differentiation by symptom severity in outpatients is plausible.

Comparisons with reports of the severe acute respiratory syndrome (SARS) in 2003 and the Middle East respiratory syndrome (MERS) are limited as mild cases of these diseases were uncommon and most patients with SARS and MERS required hospital admission (requiring intensive care in $>20 \%$ of SARS cases and $>50 \%$ of MERS cases). ${ }^{27} 28$ The sequence of symptoms we describe for COVID-19 is similar to descriptions of SARS, with initial presentation of systemic symptoms and cough, followed by lower respiratory symptoms and gastrointestinal manifestations. ${ }^{27}$ Notable differences include that fever is universal in reports of SARS $(99.9 \%)$ and upper respiratory symptoms are less common than with COVID-19 (eg, rhinorrhoea, sore throat both $<20 \%$ ). Patients with MERS also more frequently have fever and dyspnoea, with lower rates of upper respiratory symptoms. ${ }^{28}$ Gastrointestinal symptoms appear at similar rates $(20 \%-30 \%)$ in SARS and MERS, despite the overall higher severity of respiratory disease. 
Table 5 Covariates affecting duration of symptoms derived from accelerated failure time model with each symptom modelled separately

\begin{tabular}{|c|c|c|c|}
\hline Symptom & Covariate & Duration multiplier $(95 \% \mathrm{Cl})$ & $P$ value \\
\hline Abdominal pain & Immune suppression & 7.48 (3.26 to 17.18$)$ & $<0.001$ \\
\hline \multirow[t]{3}{*}{ Body aches } & Mild initial symptom severity* & Reference & \\
\hline & Moderate initial symptom severity & 1.87 (1.44 to 2.42$)$ & $<0.001$ \\
\hline & Severe initial symptom severity & 4.01 (1.66 to 9.68$)$ & 0.002 \\
\hline \multirow[t]{3}{*}{ Chest tightness } & Mild initial symptom severity & Reference & \\
\hline & Moderate initial symptom severity & 1.36 (0.96 to 1.93$)$ & 0.083 \\
\hline & Severe initial symptom severity & $4.26(1.68$ to 10.78$)$ & 0.002 \\
\hline \multirow[t]{3}{*}{ Chills } & Mild initial symptom severity & Reference & \\
\hline & Moderate initial symptom severity & 1.57 (1.21 to 2.03$)$ & 0.001 \\
\hline & Severe initial symptom severity & 2.75 (1.28 to 5.90$)$ & 0.009 \\
\hline \multirow[t]{3}{*}{ Confusion } & Race=White & Reference & \\
\hline & Race=Black & 1.65 (1.03 to 2.63$)$ & 0.036 \\
\hline & Race=Other & 7.44 (3.98 to 13.92$)$ & $<0.001$ \\
\hline \multirow[t]{4}{*}{ Congestion } & Female & 1.57 (1.12 to 2.20$)$ & 0.009 \\
\hline & Mild initial symptom severity & Reference & \\
\hline & Moderate initial symptom severity & $1.66(1.20$ to 2.31$)$ & 0.002 \\
\hline & Severe initial symptom severity & 1.97 (0.59 to 6.57$)$ & 0.27 \\
\hline \multirow[t]{3}{*}{ Cough } & Mild initial symptom severity & Reference & \\
\hline & Moderate initial symptom severity & 1.52 (1.15 to 1.99$)$ & 0.003 \\
\hline & Severe initial symptom severity & 3.46 (1.25 to 9.58$)$ & 0.017 \\
\hline \multirow[t]{6}{*}{ Diarrhoea } & Race $=$ White & Reference & \\
\hline & Race=Black & 0.56 (0.37 to 0.84$)$ & 0.005 \\
\hline & Race=Other & 0.61 (0.39 to 0.96$)$ & 0.033 \\
\hline & Mild initial symptom severity & Reference & \\
\hline & Moderate initial symptom severity & 1.72 (1.24 to 2.38$)$ & 0.001 \\
\hline & Severe initial symptom severity & 3.41 (0.91 to 12.74$)$ & 0.068 \\
\hline \multirow[t]{3}{*}{ Dizziness } & Mild initial symptom severity & Reference & \\
\hline & Moderate initial symptom severity & 1.79 (1.24 to 2.58$)$ & 0.002 \\
\hline & Severe initial symptom severity & 6.09 (1.80 to 20.54$)$ & 0.004 \\
\hline \multirow[t]{4}{*}{ Fever } & Mild initial symptom severity & Reference & \\
\hline & Moderate initial symptom severity & 1.31 (0.92 to 1.87$)$ & 0.132 \\
\hline & Severe initial symptom severity & $4.76(1.51$ to 15.07$)$ & 0.008 \\
\hline & Immune suppression & 1.97 (1.01 to 3.85$)$ & 0.048 \\
\hline \multirow[t]{4}{*}{ Headache } & Female & 1.34 (1.01 to 1.79$)$ & 0.043 \\
\hline & Mild initial symptom severity & Reference & \\
\hline & Moderate initial symptom severity & 2.10 (1.58 to 2.78$)$ & $<0.001$ \\
\hline & Severe initial symptom severity & $1.91(0.74$ to 4.98$)$ & 0.184 \\
\hline Joint pain & Obesity & 1.74 (1.09 to 2.76$)$ & 0.019 \\
\hline \multirow[t]{2}{*}{ Loss of smell or taste } & Female & 1.63 (1.24 to 2.13$)$ & $<0.001$ \\
\hline & Immune suppression & 3.06 (1.48 to 6.34$)$ & 0.003 \\
\hline \multirow[t]{3}{*}{ Nausea } & Mild initial symptom severity & Reference & \\
\hline & Moderate initial symptom severity & 1.73 (1.15 to 2.61$)$ & 0.009 \\
\hline & Severe initial symptom severity & 2.76 (0.73 to 10.42$)$ & 0.135 \\
\hline
\end{tabular}


Table 5 Continued

\begin{tabular}{|c|c|c|c|}
\hline Symptom & Covariate & Duration multiplier (95\% Cl) & $P$ value \\
\hline \multirow[t]{3}{*}{$\mathrm{SOB}$ at rest } & Race $=$ White & Reference & \\
\hline & Race=Black & 2.87 (1.63 to 5.07$)$ & $<0.001$ \\
\hline & Asthma & 1.97 (1.20 to 3.24$)$ & 0.007 \\
\hline \multirow[t]{2}{*}{ SOB with exertion } & Mild initial symptom severity & Reference & \\
\hline & Severe initial symptom severity & Undefined $†$ & $\mathrm{~N} / \mathrm{A}$ \\
\hline \multirow[t]{3}{*}{ Sore throat } & Mild initial symptom severity & Reference & \\
\hline & Moderate initial symptom severity & $1.25(0.83$ to 1.88$)$ & 0.279 \\
\hline & Severe initial symptom severity & $4.22(1.70$ to 10.47$)$ & 0.002 \\
\hline
\end{tabular}

*Initial symptom severity is the symptom severity as assessed by the provider at VOMC intake visit (see box 1) or patient-reported severity if provider-assessed severity not available.

†Unable to calculate due to small $\mathrm{N}$ with wide standard deviation for this covariate.

SOB, shortness of breath; VOMC, Virtual Outpatient Management Clinic.

\section{Strengths and limitations of the study}

Our data on the course of symptoms in outpatients are robust due to the structure of the VOMC, which was staffed to meet the anticipated 'surge' of patients in March-May 2020 and therefore had skilled providers contacting patients and completing full note templates regularly through the course of acute illness. Exclusion criteria were minimal and primarily in place to improve accuracy of symptom recollection. Missing clinical data were minimal (eg, low-risk patients contacted every 48 hours instead of 24 hours), allowing for standard approach to imputation.

The primary limitation of this study is that it represents a single-centre cohort of patients early in the SARS-CoV-2 pandemic. Testing criteria favoured the inclusion of working-age individuals in the cohort. We have limited numbers of patients with comorbidities and cannot therefore draw conclusions about the duration of symptoms related to specific conditions (eg, chronic obstructive pulmonary disease). Furthermore, we note that asymptomatic or mildly symptomatic cases would be less likely to qualify for testing at the time of this study (see criteria in box 1) and may have been less likely, even if tested, to accept a referral to VOMC (we do not have data on reasons for declined referrals). Because of the relatively small size of the cohort, we also have small numbers of less common symptoms (eg, confusion and rash).

Another limitation to the VOMC cohort data is the time delay to the VOMC intake visit. Our usual care requires a positive SARS-CoV-2 test prior to VOMC enrolment, and delays in the testing process or results notification could attenuate patient recall of initial symptoms. It is also possible that delays would reduce the intake of patients with severe symptoms (as they escalate to admission) as well as mild symptoms (as they resolve). To reduce the effects of testing delays on our study, we limited the study to patients within 10 days of symptom onset and used chart review to verify symptoms reported in the testing process.

Discharge timing in the VOMC was a limitation for our follow-up data: the VOMC discharge criteria mirrored the Centers for Disease Control and Prevention terminology of symptom 'improvement,' but not resolution. The per cent of patients still symptomatic at the last phone call varied among symptoms (table 4). We find in other work (unpublished data) that minor residual symptoms are common after VOMC discharge (reported in 55 of 158 , $34.8 \%$, of patients contacted a mean of 37.9 days after discharge $)$ and that few $(\mathrm{n}=7,4.4 \%)$ have symptoms requiring medical follow-up (eg, by a primary care physician or specialist).$^{29}$ These residual symptoms are not captured in the heat map data after their final VOMC call.

'Long COVID-19' has now been described as symptoms persisting beyond the acute illness, ${ }^{30}$ including fatigue, palpitations, 'brain fog' and other symptoms that were not known in March 2020. We have identified these symptoms in individual cases within the VOMC cohort who received prolonged care ${ }^{19}$ but did not capture these specific symptoms during the acute care described in this study.

\section{CONCLUSION}

Overall, we find that the symptom course of outpatients with COVID-19 follows a pattern described in early observations with a typical illness course progressing from early symptoms (systemic, upper respiratory and cough) to lower respiratory and gastrointestinal symptoms. We confirm that symptoms of altered smell or taste and headache are common in outpatients. Prolonged symptoms are common and the severity of symptoms in the acute 
phase of illness is the most significant predictor of disease duration.

Acknowledgements We would like to acknowledge Dr David Roberts for the design of the structured intake assessment note and nurse follow-up notes. We would also like to acknowledge the members of the Virtual Outpatient Management Clinic including faculty, staff and administrative members of the Paul W Seavey Comprehensive Internal Medicine Clinic and Emory at Rockbridge Primary Care Clinic as well as the physicians, nurses and advanced practice providers who volunteered from other sites.

Contributors DCT had full access to all the data in the study and takes responsibility for the integrity of the data and the accuracy of the data analysis. Transparency statement-DT attests that the manuscript is an honest, accurate and transparent account of the study being reported; that no important aspects of the study have been omitted; and that any discrepancies from the study as originally planned have been explained. Concept and design-JBO, GDO and DCT. Acquisition, analysis or interpretation of data-JBO, EJT, GDO and DCT. Drafting of the manuscript-JBO, EJT and DCT. Critical revision of the manuscript for important intellectual content-JBO, EJT, GDO and DCT. Statistical analysis-DCT. Obtained funding - JBO

Funding JB0 is funded by the Georgia Geriatrics Workforce Enhancement Program (GA-GWEP) COVID-19 Telehealth award, supported by the Health Resources and Services Administration (HRSA) of the US Department of Health and Human Services (HHS) as part of Award Number T1MHP39056 totalling \$90 625 with 0\% financed with non-governmental sources.

Dissemination declaration We plan to disseminate our results publicly but it would not be feasible to reach discharged patients with study results and therefore we do not plan to contact study participants for dissemination.

Disclaimer The contents are those of the author and do not necessarily represent the official views of, nor an endorsement, by HRSA, HHS or the US government. The funder/sponsor had no direct role in study planning and conduct, reporting or authorship. The Corresponding Author has the right to grant on behalf of all authors and does grant on behalf of all authors, a worldwide licence to the Publishers and its licensees in perpetuity, in all forms, formats and media (whether known now or created in the future), to (1) publish, reproduce, distribute, display and store the Contribution, (2) translate the Contribution into other languages, create adaptations, reprints, include within collections and create summaries, extracts and/or abstracts of the Contribution, (3) create any other derivative work(s) based on the Contribution, (4) to exploit all subsidiary rights in the Contribution, (5) the inclusion of electronic links from the Contribution to third party material wherever it may be located; and, (6) license any third party to do any or all of the above.

Competing interests All authors have completed the Unified Competing Interest form (available on request from the corresponding author) and declare: no support from any organisation for the submitted work; no financial relationships with any organisations that might have an interest in the submitted work in the previous 3 years, no other relationships or activities that could appear to have influenced the submitted work. GDO served on an advisory board of Eyepoint Pharmaceuticals in 2019. It is unrelated to the current work.

Patient consent for publication Not required.

Ethics approval The study was approved by the Emory University Institutional Review Board (STUDY00000766), which granted both a waiver of informed consent and a waiver of the Health Information Portability and Privacy Act as the study posed no more than minimal risk.

Provenance and peer review Not commissioned; externally peer reviewed.

Data availability statement Data are available upon reasonable request. Deidentified data are available for sharing upon reasonable request to DCT (ORCID 0000-0001-9761-6124). Data include patient demographics and comorbidities as well as symptom dates for all participants.

Supplemental material This content has been supplied by the author(s). It has not been vetted by BMJ Publishing Group Limited (BMJ) and may not have been peer-reviewed. Any opinions or recommendations discussed are solely those of the author(s) and are not endorsed by BMJ. BMJ disclaims all liability and responsibility arising from any reliance placed on the content. Where the content includes any translated material, BMJ does not warrant the accuracy and reliability of the translations (including but not limited to local regulations, clinical guidelines, terminology, drug names and drug dosages), and is not responsible for any error and/or omissions arising from translation and adaptation or otherwise.
Open access This is an open access article distributed in accordance with the Creative Commons Attribution Non Commercial (CC BY-NC 4.0) license, which permits others to distribute, remix, adapt, build upon this work non-commercially, and license their derivative works on different terms, provided the original work is properly cited, appropriate credit is given, any changes made indicated, and the use is non-commercial. See: http://creativecommons.org/licenses/by-nc/4.0/.

\section{ORCID iDs}

James B O'Keefe http://orcid.org/0000-0002-7151-7184

Elizabeth J Tong http://orcid.org/0000-0001-7724-5899

Ghazala D 0'Keefe http://orcid.org/0000-0002-5641-4195

David C Tong http://orcid.org/0000-0001-9761-6124

\section{REFERENCES}

1 Argenziano MG, Bruce SL, Slater CL, et al. Characterization and clinical course of 1000 patients with coronavirus disease 2019 in New York: retrospective case series. BMJ 2020;369:m1996.

2 Docherty AB, Harrison EM, Green CA, et al. Features of 20133 UK patients in hospital with covid-19 using the ISARIC WHO Clinical Characterisation Protocol: prospective observational cohort study. BMJ 2020;369:m1985.

3 Huang C, Wang Y, Li X, et al. Clinical features of patients infected with 2019 novel coronavirus in Wuhan, China. Lancet 2020;395:497-506

4 Wang D, Hu B, Hu C, et al. Clinical characteristics of 138 hospitalized patients with 2019 novel coronavirus-infected pneumonia in Wuhan, China. JAMA 2020;323:1061-9.

5 Zhou F, Yu T, Du R, et al. Clinical course and risk factors for mortality of adult inpatients with COVID-19 in Wuhan, China: a retrospective cohort study. Lancet 2020;395:1054-62.

6 Stokes EK, Zambrano LD, Anderson KN, et al. Coronavirus Disease 2019 Case Surveillance - United States, January 22-May 30, 2020. MMWR Morb Mortal Wkly Rep 2020;69:759-65.

7 Centers for Disease Control and Prevention. Interim clinical guidance for management of patients with confirmed coronavirus disease (COVID-19), 2020. Available: https://www.cdc.gov/coronavirus/2019ncov/hcp/clinical-guidance-management-patients.html

8 World Health Organization. Home care for patients with COVID-19 presenting with mild symptoms and management of their contacts, 2020. Available: https://www.who.int/publications-detail/homecare-for-patients-with-suspected-novel-coronavirus-(ncov)infection-presenting-with-mild-symptoms-and-management-ofcontacts

9 Pullen MF, Skipper CP, Hullsiek KH, et al. Symptoms of COVID-19 outpatients in the United States. Open Forum Infect Dis 2020;7:ofaa271.

10 Lapostolle F, Schneider E, Vianu I, et al. Clinical features of 1487 COVID-19 patients with outpatient management in the greater Paris: the COVID-call study. Intern Emerg Med 2020;15:813-7.

11 Kluytmans-van den Bergh MFQ, Buiting AGM, Pas SD, et al. Prevalence and clinical presentation of health care workers with symptoms of coronavirus disease 2019 in 2 Dutch hospitals during an early phase of the pandemic. JAMA Netw Open 2020;3:e209673.

12 Kim G-u, Kim M-J, Ra SH. Clinical characteristics of asymptomatic and symptomatic patients with mild COVID-19. Clinical Microbiology and Infection 2020;26:948.e1-948.e3.

13 Killerby ME, Link-Gelles R, Haight SC, et al. Characteristics associated with hospitalization among patients with COVID-19 - metropolitan Atlanta, Georgia, March-April 2020. MMWR Morb Mortal Wkly Rep 2020;69:790-4.

14 Grant MC, Geoghegan L, Arbyn M, et al. The prevalence of symptoms in 24,410 adults infected by the novel coronavirus (SARSCoV-2; COVID-19): a systematic review and meta-analysis of 148 studies from 9 countries. PLoS One 2020;15:e0234765-19.

15 Burke RM, Killerby ME, Newton S, et al. Symptom Profiles of a Convenience Sample of Patients with COVID-19 - United States, January-April 2020. MMWR Morb Mortal Wkly Rep 2020;69:904-8.

16 Tabata S, Imai K, Kawano S, et al. Clinical characteristics of COVID-19 in 104 people with SARS-CoV-2 infection on the diamond Princess cruise SHIP: a retrospective analysis. Lancet Infect Dis 2020;20:1043-50.

17 Tenforde MW, Kim SS, Lindsell CJ, et al. Symptom duration and risk factors for delayed return to usual health among outpatients with COVID-19 in a multistate health care systems network United States, March-June 2020. MMWR Morb Mortal Wkly Rep 2020;69:993-8. 
18 Nehme M, Braillard O, Alcoba G, et al. COVID-19 symptoms: longitudinal evolution and persistence in outpatient settings. Ann Intern Med 2020:M20-5926.

19 Cellai M, O'Keefe JB. Characterization of prolonged COVID-19 symptoms in an outpatient telemedicine clinic. Open Forum Infectious Diseases 2020;7:813-4.

20 O'Keefe JB, Tong EJ, Taylor TH. Use of a Telemedicine Risk Assessment Tool to Predict the Risk of Hospitalization of 496 Outpatients with COVID-19: Retrospective Analysis. JMIR Public Health Surveill (forthcoming)tool

21 Ramakrishnan A, Zreloff J, Moore M, et al. Prolonged symptoms after COVID-19 infection in outpatients. Open Forum Infectious Diseases. In Press.

22 Dey T, Mukherjee A, Chakraborty S. A Practical Overview and Reporting Strategies for Statistical Analysis of Survival Studies. Chest 2020;158:S39-48.

23 Heymann DL, Shindo N. Who scientific and technical Advisory group for infectious hazards COVID-19: what is next for public health? Lancet 2020;395:542-5.
24 Cohen PA, Hall LE, John JN, et al. The early natural history of SARSCoV-2 infection: clinical observations from an urban, ambulatory COVID-19 clinic. Mayo Clin Proc 2020;95:1124-6.

25 Prezant DJ, Zeig-Owens R, Schwartz T, et al. Medical leave associated with COVID-19 among emergency medical system responders and firefighters in New York City. JAMA Netw Open 2020;3:e2016094-4.

26 Carfi A, Bernabei R, Landi F, et al. Persistent symptoms in patients after acute COVID-19. JAMA 2020;324:603.

27 Peiris JSM, Yuen KY, Osterhaus ADME, et al. The severe acute respiratory syndrome. N Engl J Med 2003;349:2431-41.

28 Arabi YM, Balkhy HH, Hayden FG, et al. Middle East respiratory syndrome. N Engl J Med 2017;376:584-94.

29 O'Keefe JB, Datoo O'Keefe GA, Mufarreh A. Short paper: risk factors for long-term persistent symptoms in COVID-19 in an outpatient cohort 2020.

30 Greenhalgh T, Knight M, A'Court C, A'Court C, et al. Management of post-acute covid-19 in primary care. BMJ 2020;370:m3026-8. 